\section{Circle of Willis}

Elliot J. Roth

Physical Medicine and Rehabilitation, Northwestern University, Feinberg School of Medicine, Chicago, IL, USA

\section{Definition}

The circle of Willis is the anatomical name given to the formation of arteries at the base of the brain that contribute the overwhelming majority of blood supply to the brain.

\section{Current Knowledge}

The circle of Willis is formed by the connections between the predominantly horizontal branches that derive from the middle cerebral arteries anteriorly and from the basilar artery posteriorly. The right and left middle cerebral arteries each give off an anterior cerebral artery (forming the anterolateral borders of the circle of Willis), which goes forward to supply blood to the frontal lobe. These anterior cerebral arteries are connected to each other by the anterior communicating artery, which forms the front of the circle. Posteriorly, the basilar artery bifurcates into the right and left posterior cerebral arteries, which supply the occipital and posterior temporal lobes and the cerebellum, forming the posterior border of the circle. Each posterior cerebral artery is connected to the middle cerebral artery on its corresponding side by a posterior communicating artery, forming the posterolateral borders of the circle. There are several clinical implications of the circle pattern of these arteries. Perhaps most importantly, because of the interconnectedness of the arteries that result from this circle format, if one of the main arteries is occluded, the distal smaller arteries that it supplies can potentially receive blood from the other arteries that make up the circle, a phenomenon known as collateral circulation. This helps to prevent cerebral ischemia and stroke. The circle of Willis also is a common site for cerebral aneurysms, with the greatest numbers involving the anterior communicating artery, posterior communicating arteries, and middle cerebral arteries.

\section{Cross-References}

- Anterior Cerebral Artery

- Anterior Communicating Artery

- Basilar Artery

- Internal Carotid Artery

- Middle Cerebral Artery

- Posterior Cerebral Artery

- Posterior Communicating Artery

- Vertebrobasilar System 